PROCEEDINGS OF THE

AMERICAN MATHEMATICAL SOCIETY

Volume 136, Number 9, September 2008, Pages 3171-3176

S 0002-9939(08)09460-4

Article electronically published on April 30, 2008

\title{
A NOTE ON GENERATING FUNCTIONS FOR HAUSDORFF MOMENT SEQUENCES
}

\author{
OLIVER ROTH, STEPHAN RUSCHEWEYH, AND LUIS SALINAS
}

(Communicated by Andreas Seeger)

\begin{abstract}
For functions $f$ whose Taylor coefficients at the origin form a Hausdorff moment sequence we study the behaviour of $w(y):=|f(\gamma+i y)|$ for $y>0(\gamma \leq 1$ fixed $)$.
\end{abstract}

\section{INTRODUCTION AND STATEMENT OF THE RESULTS}

A sequence $\left\{a_{k}\right\}_{k \geq 0}$ of non-negative real numbers, $a_{0}=1$, is called a Hausdorff moment sequence if there is a probability measure 11 on $[0,1]$ such that

$$
a_{k}=\int_{0}^{1} t^{k} d \mu(t), \quad k \geq 0,
$$

or, equivalently,

$$
F(z)=\sum_{k=0}^{\infty} a_{k} z^{k}=\int_{0}^{1} \frac{d \mu(t)}{1-t z},
$$

and $F$ is its generating function.

It is well known (Hausdorff [2]) that a sequence $\left\{a_{k}\right\}_{k \geq 0}$ with $a_{0}=1$ is a Hausdorff moment sequence if and only if it is completely monotone, i.e.

$$
\Delta^{n} a_{k}:=\Delta^{n-1} a_{k}-\Delta^{n-1} a_{k+1} \geq 0, \quad k \geq 0, n \geq 1,
$$

where $\Delta^{0}$ is the identity operator: $\Delta^{0} a=a$.

Let $\mathcal{T}$ denote the set of such generating functions $F$. They are analytic in the slit domain $\Lambda:=\mathbb{C} \backslash[1, \infty)$ and also belong to the set of Pick functions $P(-\infty, 1)$ (see Donoghue [1] for more information on Pick functions).

Wirths [5] has shown that $f \in \mathcal{T}$ implies that the function $z f(z)$ is univalent in the half-plane $\operatorname{Re} z<1$, and recently the theory of universally prestarlike mappings

Received by the editors July 5, 2007.

2000 Mathematics Subject Classification. Primary 30E05, 26 A48.

Key words and phrases. Hausdorff moment sequences, completely monotone sequences, Pick functions, convolution, polylogarithms.

The first and second authors acknowledge partial support from the German-Israeli Foundation (grant G-809-234.6/2003). The second and third authors received partial support from FONDECYT (grants 1070269 and 7070131) and DGIP-UTFSM (grant 240721).

${ }^{1}$ Here and in the sequel we always assume that the measures are Borel.

(C)2008 American Mathematical Society Reverts to public domain 28 years from publication 
has been developed, showing a close link to $\mathcal{T}$; see 4 . Many classical functions belong to $\mathcal{T}$ or are closely related to it. We mention only the polylogarithms

$$
L i_{\alpha}(z):=\sum_{k=1}^{\infty} \frac{z^{k}}{k^{\alpha}}, \quad \alpha \geq 0
$$

where $L i_{\alpha}(z) / z \in \mathcal{T}$ and which we are going to study somewhat closer in the sequel.

The main result in this paper is

Theorem 1.1. For $f \in \mathcal{T}$ we have

$$
\operatorname{Re} \frac{f\left(\gamma+i y_{1}\right)}{f\left(\gamma+i y_{2}\right)} \geq 1, \quad \gamma \in(-\infty, 1], 0<y_{1} \leq y_{2}
$$

This relation does not hold, in general, for $\gamma>1$.

Theorem 1.1 has the following immediate consequence.

Corollary 1.2. For $f \in \mathcal{T}$ and $\gamma \in(-\infty, 1]$ fixed, the function $|f(\gamma+i y)|$ is monotonically decreasing with $y>0$ increasing.

In the case $\gamma=0$ Theorem 1.1 admits a slight generalization. It is well-known and easy to verify that $\mathcal{T}$ is invariant under the Hadamard product: if

$$
f(z)=\sum_{k=0}^{\infty} a_{k} z^{k}, \in \mathcal{T}, \quad g(z)=\sum_{k=0}^{\infty} b_{k} z^{k} \in \mathcal{T},
$$

then also

$$
(f * g)(z):=\sum_{k=0}^{\infty} a_{k} b_{k} z^{k} \in \mathcal{T} .
$$

Theorem 1.3. For $f, g \in \mathcal{T}$ we have

$$
\operatorname{Re} \frac{(f * g)(i y)}{f(i y)} \geq 1, \quad y>0 .
$$

Therefore, under the same assumption,

$$
|f(i y)| \leq|(f * g)(i y)|, \quad y>0 .
$$

For the polylogarithms and $0<\alpha \leq \beta$ it is clear that $L i_{\beta}=L i_{\alpha} * L i_{\beta-\alpha}$, so that we get

Corollary 1.4. For $0 \leq \alpha<\beta$

$$
\left|L i_{\alpha}(i y)\right| \leq\left|L i_{\beta}(i y)\right|, \quad y>0 .
$$

This result can also be obtained and even strengthened using Corollary 1.2 and the deeper relation

$$
\frac{L i_{\alpha}}{L i_{\beta}} \in \mathcal{T}, \quad 0 \leq \alpha \leq \beta,
$$

recently established in [4].

For a certain subset of $\mathcal{T}$ we can go one step beyond Corollary 1.2 as far as the behaviour of $|f(i y)|$ for $y>0$ is concerned. 
Theorem 1.5. Let

$$
f(z)=\int_{0}^{1} \frac{\sigma(t) d t}{1-t z}
$$

where $\sigma \in \mathcal{C}^{1}((0,1))$ is positive and with $t \sigma^{\prime}(t) / \sigma(t)$ decreasing. Then, for $w(y):=$ $|f(i y)|$, the function $y w^{\prime}(y) / w(y)$ decreases with $y>0$ increasing.

Fundamental for the proof of Theorem 1.5 is the following result, which is based on a general theorem in 4 .

Theorem 1.6. Let $f$ be as in Theorem 1.5. Then, for $x \in[0,1]$,

$$
\frac{f(z)}{f(x z)} \in \mathcal{T} \text {. }
$$

One can show that the conclusion of Theorem 1.6 is not generally valid for $f \in \mathcal{T}$. However, for the functions $g_{\alpha}(z):=\frac{1}{z} L i_{\alpha}(z), \alpha>0$, we have

$$
g_{\alpha}(z)=\frac{1}{\Gamma(a)} \int_{0}^{1} \frac{\log ^{\alpha-1}(1 / t)}{1-t z} d t
$$

for which the assumptions of Theorem 1.5 are fulfilled. Thus both Theorem 1.5 and Theorem 1.6 apply to $g_{\alpha}$.

\section{Proofs}

We first note that the convex set $\mathcal{T}$ satisfies the condition of the main theorem in [3], which for the present case can be stated as follows:

Lemma 2.1. Let $\lambda_{1}, \lambda_{2}$ be two continuous linear functionals on $\mathcal{T}$ and assume that $0 \notin \lambda_{2}(\mathcal{T})$. Then the range of the functional

$$
\lambda(f):=\frac{\lambda_{1}(f)}{\lambda_{2}(f)}
$$

over $\mathcal{T}$ equals the set

$$
\left\{\lambda\left(\frac{\rho}{1-t_{1} z}+\frac{1-\rho}{1-t_{2} z}\right): \rho, t_{1}, t_{2} \in[0,1]\right\} .
$$

Proof of Theorem 1.1. First we note that it is enough to prove (1.1) for $\gamma=1$ only. This is because $f \in \mathcal{T}$ implies $f(z-\delta) / f(-\delta) \in \mathcal{T}$ for all $\delta>0$. In Lemma 2.1] we choose $\lambda_{j}(f):=f\left(1+i y_{j}\right), j=1,2$. Since $\operatorname{Im} f(z)>0$ for $f \in \mathcal{T}$ and $\operatorname{Im} z>0$, it is clear that $0 \notin \lambda_{2}(\mathcal{T})$. Lemma 2.1 now implies that for the proof of Theorem 1.1 we only need to show that the expression

$$
\frac{\frac{\rho}{1-t_{1}-i t_{1} y_{1}}+\frac{1-\rho}{1-t_{2}-i t_{2} y_{1}}}{\frac{\rho}{1-t_{1}-i t_{1} y_{2}}+\frac{1-\rho}{1-t_{2}-i t_{2} y_{2}}}, \quad \rho, t_{1}, t_{2} \in[0,1],
$$

is located in the half-plane $\{w: \operatorname{Re} w \geq 1\}$. To simplify this expression we set $\kappa:=(1-\rho) / \rho, \tau:=y_{1} / y_{2}$. Then our claim is

$$
\operatorname{Re} q\left(\kappa, y, \tau, t_{1}, t_{2}\right) \geq 1, \quad \kappa \geq 0, y>0, t_{1}, t_{2}, \tau \in[0,1],
$$


where

$$
q\left(\kappa, y, \tau, t_{1}, t_{2}\right)=\frac{\frac{1}{1-t_{1}-i \tau y t_{1}}+\frac{\kappa}{1-t_{2}-i \tau y t_{2}}}{\frac{1}{1-t_{1}-i y t_{1}}+\frac{\kappa}{1-t_{2}-i y t_{2}}} .
$$

Note that by symmetry we may assume that $t_{1} \leq t_{2}$. For fixed $y, \tau, t_{1}, t_{2}$ the values of $w(\kappa):=q\left(\kappa, y, \tau, t_{1}, t_{2}\right), \kappa \geq 0$, form a circular arc connecting the points $w(0)=v\left(t_{1}\right)$ and $w(\infty)=v\left(t_{2}\right)$, where

$$
v(t)=\frac{1-t-i y t}{1-t-i \tau y t} .
$$

It is easily checked that under our assumptions for $y$ and $\tau$ the function $\operatorname{Re} v(t)$ increases with $t \in[0,1]$ and, in particular, $\operatorname{Re} v(t) \geq \operatorname{Re} v(0)=1$. This implies that

$$
1 \leq \operatorname{Re} w(0) \leq \operatorname{Re} w(\infty) .
$$

We will prove that $\operatorname{Re} w^{\prime}(0) \geq 0$. Once this is done a simple geometric consideration shows that under these circumstances the circular arc $w(\kappa), \kappa \geq 0$, cannot leave the half-plane $\{w: \operatorname{Re} w \geq 1\}$, which then completes the proof of (1.1).

Calculation yields

$$
\operatorname{Re} w^{\prime}(0)=(1-\tau)\left(t_{2}-t_{1}\right) y^{2} \frac{Z}{N}
$$

where

$$
\begin{aligned}
Z & =t_{1}^{*} t_{2}^{*}\left(t_{2}-t_{1}\right)+\left(t_{1} t_{2}^{*}+t_{2} t_{1}^{*}\right) t_{1}^{*} t_{2}^{*} \tau+t_{1} t_{2} y^{2} \tau\left(t_{1} t_{2}^{*}+t_{2} t_{1}^{*}-\tau\left(t_{2}-t_{1}\right)\right), \\
N & =\left(\left(1-t_{1}\right)^{2}+\left(t_{1} y \tau\right)^{2}\right)\left(\left(1-t_{2}\right)^{2}+\left(t_{2} y \tau\right)^{2}\right)\left(\left(1-t_{2}\right)^{2}+\left(t_{2} y\right)^{2}\right),
\end{aligned}
$$

and $t_{j}^{*}:=1-t_{j}$. Here all terms are non-negative (note that

$$
s(\tau):=t_{1} t_{2}^{*}+t_{2} t_{1}^{*}-\tau\left(t_{2}-t_{1}\right)
$$

decreases with $\tau$ and is therefore not smaller than $\left.s(1)=2 t_{1} t_{2}^{*} \geq 0\right)$.

It remains to show that (1.1) does not hold, in general, for $\gamma>1$. Let $\gamma=$ $1+\varepsilon, \varepsilon>0$, and choose

$$
f(z):=\frac{1}{1+2 \varepsilon}+\frac{2 \varepsilon}{1+2 \varepsilon} \frac{1}{1-z} \in \mathcal{T} .
$$

Then, using $y_{1}=\varepsilon, y_{2}=1$,

$$
\operatorname{Re} \frac{f(\gamma+i \varepsilon)}{f(\gamma+i)}=\frac{2 \varepsilon}{1+\varepsilon^{2}}<1
$$

Proof of Theorem 1.3. If

$$
g(z)=\int_{0}^{1} \frac{d \mu(t)}{1-t z}
$$

then

$$
\frac{(f * g)(i y)}{f(i y)}=\int_{0}^{1} \frac{f(i t y)}{f(i y)} d \mu(t),
$$

which is a convex combination of the values of $f(i t y) / f(i y)$. By Theorem 1.1 these are all in the half-plane $\{w: \operatorname{Re} w \geq 1\}$.

For the proof of Theorem 1.6 we need the following result from [4]. 
Lemma 2.2. Let $f, g \in \mathcal{T}$ be represented by

$$
f(z)=\int_{0}^{1} \frac{\varphi(t) d t}{1-t z}, \quad g(z)=\int_{0}^{1} \frac{\psi(t) d t}{1-t z}
$$

with non-negative Borel functions $\varphi, \psi$ on $(0,1)$. If $\varphi(t) \psi(s) \geq \varphi(s) \psi(t)$ holds for all $0<s<t<1$, then $f / g \in \mathcal{T}$.

Proof of Theorem 1.6. We have

$$
f(x z)=\int_{0}^{1} \frac{\sigma(t) d t}{1-t x z}=\int_{0}^{1} \frac{\sigma^{*}(t) d t}{1-t z},
$$

with

$$
\sigma^{*}(t):= \begin{cases}\frac{1}{x} \sigma(t / x), & 0<t \leq x, \\ 0, & x<t<1 .\end{cases}
$$

The condition

$$
\sigma(t) \sigma^{*}(s) \geq \sigma(s) \sigma^{*}(t), \quad 0<s<t<1,
$$

is immediately fulfilled if $t>x$. Otherwise we are left with

$$
\sigma(t) \sigma(s / x) \geq \sigma(s) \sigma(t / x), \quad 0<s<t \leq x .
$$

This requires that $\sigma(t) / \sigma(t / x)$ increases with $t$. Taking logarithms and differentiating w.r.t. the variable $t$, we find as a necessary and sufficient condition for (2.1) that $t \sigma^{\prime}(t) / \sigma(t)$ decreases for $t$ increasing. The result now follows from Lemma 2.2 ,

Proof of Theorem 1.5. We apply Theorem 1.1 to the function $F$ of Theorem 1.6 . Then, for $x, \tau \in(0,1)$, we get

$$
\left|\frac{f(i y \tau) f(i y x)}{f(i y x \tau) f(i y)}\right| \geq 1, \quad y>0 .
$$

Taking logarithms we obtain

$$
(\log w(y)-\log w(x y))-(\log w(\tau y)-\log w(x \tau y)) \leq 0 .
$$

Dividing by $1-x$ and letting $x \rightarrow 1-0$ yield

$$
\frac{y w^{\prime}(y)}{w(y)} \leq \frac{\tau y w^{\prime}(\tau y)}{w(\tau y)}
$$

which implies the assertion.

\section{REFERENCES}

[1] W.B. Donoghue Jr., Monotone matrix functions and analytic continuation, Grundlehren Math. Wiss. 207, Springer-Verlag (1974). MR0486556 (58:6279)

[2] F. Hausdorff, Summationsmethoden und Momentfolgen I, Math. Z. 9 (1921), 74-109. MR.1544453

[3] S. Ruscheweyh, Nichtlineare Extremalprobleme für holomorphe Stieltjesintegrale, Math. Z. 142 (1975), 19-23. MR0374406 (51:10606) 
[4] S. Ruscheweyh, L. Salinas, and T. Sugawa, Completely monotone sequences and universally prestarlike functions, preprint 2007.

[5] K.J. Wirths, Über totalmonotone Zahlenfolgen, Arch. Math. 26 (1975), 508-517. MR0396930 $(53: 790)$

Mathematisches Institut, Universität Würzburg, D-97074 Würzburg, Germany

E-mail address: roth@mathematik.uni-wuerzburg.de

Mathematisches Institut, Universität Würzburg, D-97074 Würzburg, Germany

E-mail address: ruscheweyh@mathematik.uni-wuerzburg.de

Departamento de Informática, Universidad Técnica F. Santa María, Valparaíso, ChILE

E-mail address: lsalinas@inf.utfsm.cl 